\title{
Comparison of clinical value of dynamic contrast-enhanced MRI and SPECT renal dynamic imaging of GFR measurement in the evaluation of renal function in renal transplantation
}

\author{
Hong G, Dan L, Yunhe L \\ Nuclear Medicine Department, the Third Affiliated Hospital of Inner Mongolia Medical University, Baotou, Inner Mongolia \\ Autonomous Region, China
}

Received: April 23, 2019

Accepted: May 30, 2019

Online Published: June 10, 2019

DOI: $10.5430 /$ dcc.v6n2p7

URL: https://doi.org/10.5430/dcc.v6n2p7

\begin{abstract}
Objective: To compare the clinical value of dynamic contrast-enhanced MRI (DCE-MRI) and single-photon emission computed tomography (SPECT) renal dynamic imaging in the measurement of glomerular filtration rate (GFR) in the evaluation of renal function in renal transplantation.

Methods: A total of 70 recipients who underwent renal transplantation in Baogang Hospital of Inner Mongolia from April of 2015 to April of 2018 were selected as research objects. GFR was measured in renal transplant recipients by use of DCE-MRI and SPECT (GFR-MRI and GFR-SPECT respectively), and was compared with creatinine clearance rate (Ccr). The safety of contrast media was evaluated in DCE-MRI detection.

Results: The bias of GFR-MRI against Ccr value was higher than that of GFR-SPECT against Ccr value, with $30 \%$ and $50 \%$ accuracy of GFR-MRI higher than that of GFR-SPECT, and the difference was statistically significant $(p<.05)$. Pearson correlation analysis showed that GFR-MRI and GFR-SPECT values were positively correlated to $\operatorname{Ccr}(p<.05)$, and the correlation coefficient of GFR-MRI and Ccr was higher than that of GFR-SPECT and Ccr, with the difference statistically significant $(p<$ .05). By Bland-Altman analysis, $95 \%$ confidence interval of GFR-SPECT was $95.49 \mathrm{ml} /\left(\mathrm{min} \cdot 1.73 \mathrm{~m}^{2}\right)$, and $95 \%$ confidence interval of GFR-MRI was $62.35 \mathrm{ml} /\left(\mathrm{min} \cdot 1.73 \mathrm{~m}^{2}\right)$, which was much narrower. Only 2 cases of patients developed mild rash among 70 cases of patients, and recovered spontaneously without any treatment.

Conclusions: Compared with SPECT, the bias of GFR measured by DCE-MRI against Ccr is much greater. However, DCE-MRI has a higher accuracy, correlation and consistency in comparison with Ccr, and it has a narrower confidence interval. DCE-MRI can more accurately evaluate renal function in renal transplantation by measuring GFR, and it has a high safety.
\end{abstract}

Key Words: Renal transplantation, Dynamic contrast-enhanced MRI, Single-photon emission computed tomography, Renal dynamic imaging

*Correspondence: Hong G; Email: nmgbgyyyy@163.com; Address: Nuclear Medicine Department, the Third Affiliated Hospital of Inner Mongolia Medical University, Baotou, Inner Mongolia Autonomous Region 014010, China. 


\section{INTRODUCTION}

Currently, renal transplantation has become the most effective treatment measure for end-stage renal disease, while surveillance of renal function and timely evaluation of the anatomical structure of transplant kidney are critical to effectively prolong the survival time of transplant kidney. ${ }^{[1]}$ Glomerular filtration rate (GFR) is the most direct indicator which can reflect renal filtering function, and it is also one of the most sensitive indicators which are used to evaluate renal function. ${ }^{[2]}$ However, GFR can be only acquired indirectly by calculating the clearance rate of some markers instead of being measured directly. Inulin clearance rate is a generallyacknowledged golden standard in GFR measurement, but it is not suitable to be popularized in clinical practice due to its complicated operation and time waste. ${ }^{[3]}$ Single-photon emission computed tomography (SPECT) renal dynamic imaging is a conventional examination method used to evaluate GFR in renal transplantation donors currently. Characterized by easy operation, safety and non-invasiveness, it can directly reflect GFR and urinary excretion performance. However, SPECT is not able to present the anatomical structure of blood vessels and the urinary tract in the kidney. ${ }^{[4]}$ Nuclear magnetic resonance imaging (MRI) is commonly used to understand renal structure. Meanwhile, dynamic contrastenhanced MRI (DCE-MRI) has been gradually applied to the evaluation of GFR. ${ }^{[5]}$ However, there are few researches on the accuracy and safety of DCR-MRI in the evaluation of GFR of transplant kidney after renal transplantation. 70 cases of patients with good and stable renal function were selected as research objects in this study, in order to make a comparison in the accuracy and safety between DCE-MRI and SPECT in GFR measurement of transplant kidney. It was reported as follows.

\section{DATA AND METHODS}

\subsection{General information}

A total of 70 recipients who underwent renal transplantation in Baogang Hospital of Inner Mongolia (hereinafter referred to as "our hospital") from April of 2015 to April of 2018 were selected as research objects. Among 70 cases, there were 41 cases of male patients and 29 cases of female patients, aged 16 to 62 , with an average age of (45.36 18.68$)$; weighed 36 to $87 \mathrm{~kg}$, with an average weight of $(61.23 \pm 11.06) \mathrm{kg}$; body surface area was 1.48 to $3.16 \mathrm{~m}^{2}$, and the mean value was $(2.06 \pm 0.35) \mathrm{m}^{2}$; serum creatinine level was 57.23 to $186.20 \mu \mathrm{mol} / \mathrm{L}$, and the mean value was $(94.36 \pm 26.03)$ $\mu \mathrm{mol} / \mathrm{L}$. Inclusion criteria were as follows: 1) All patients had underwent renal transplantation; 2) patients who had a good and stable renal function; 3) patients who followed the doctor's advice and actively cooperated with doctors during treatment; 4) patients who were acknowledged and required to sign informed consent forms approved and implemented by Ethics Committee in our hospital. Exclusion criteria were as follows: 1) patients who had an allergic history in contrast media; 2) patients who were allergic to the medicine used in this research; 3) patients who were with other types of serious diseases; 4) patients who had cardiac, hepatic and splenic insufficiency.

\subsection{Immunotherapy}

All immunotherapies given to patients were triplex immunosuppressive regimens: Cyclosporin A (NCPC, SFDA Approval Number: H10960009), the dose of which was 6-8 $\mathrm{mg} /(\mathrm{kg} \cdot \mathrm{d})$ initially and adjusted according to plasma concentration and serum creatinine level; Leflunomide (Suzhou Changzheng-Cinkate Pharmaceutical Co., Ltd, SFDA Approval Number: H20000550), the dose of which was $50 \mathrm{mg} / \mathrm{d}$ and then adjusted to $20 \mathrm{mg} / \mathrm{d}$ (weight $<60 \mathrm{~kg}$ ) or $30 \mathrm{mg} / \mathrm{d}$ (weight $>60 \mathrm{~kg}$ ) after 3-5 d; Mycophenolic acid (Jiangsu Stru Chem Co., Ltd, SFDA Approval Number: H12020809), the dose of which was $1.0 \mathrm{~g} / \mathrm{d}$ (weight $<60 \mathrm{~kg}$ ) or $1.5 \mathrm{~g} / \mathrm{d}$ (weight $>60 \mathrm{~kg}$ ).

\subsection{The measurement of creatinine clearance rate (Ccr)}

Before examination, all subjects were required to keep a low-protein diet with no meat and avoid strenuous exercise. It was also required to check the blood sample and 24-h urine taken on the day of measurement. Urine creatinine level and serum creatinine level were measured to calculate Ccr according to the following formula: $\mathrm{Ccr}=($ Urine creatinine level/serum creatinine level) $\times$ urine volume per minute. Ccr was corrected by body surface area (BSA), i.e., the corrected $\mathrm{Ccr}=($ Urine creatinine level/serum creatinine level $)$ $\times$ urine volume per minute $\times 1.73 / \mathrm{BSA}$, and the unit was $\mathrm{ml} /\left(\min \cdot 1.73 \mathrm{~m}^{2}\right)$.

\subsection{The measurement of GFR by DCE-MRI}

GE Discovery 750 3.0T scanner was used to perform the abdominal MRI scan to all patients. After positioning scan, 3D coronal liver acceleration volume acquisition sequence was used to perform a scan to renal regions. In the enhanced examination, double-cylinder high pressure syringe was used to make an intravenous injection on the elbow to give $0.04 \mathrm{mmol} / \mathrm{kg}$ of Gd-DOTA $(0.5 \mathrm{mmol} / \mathrm{L}$, Bayer Schering, Germany, Batch Number: 20150402) diluted with normal saline, and the injection speed was $3.0 \mathrm{ml} / \mathrm{s}$. Flushing was made by use of $10 \mathrm{ml}$ normal saline after injection. LAVA sequence scanning parameters were set as follows: TR $4.7 \mathrm{~ms}$, TE $1.9 \mathrm{~ms}$, TI $5.0 \mathrm{~ms}$, rotation angle $15^{\circ}$, pixel bandwidth $977 \mathrm{kHz}$, matrix $256 \times 160$, visual field $35.0 \mathrm{~cm} \times$ $35.0 \mathrm{~cm}$, slice thickness $2.8 \mathrm{~mm}$, spacing $2.8 \mathrm{~mm}$. 24 slices 
were acquired, and the acquisition time was $3.0 \mathrm{~s}$. Patients were required to hold their breath for consecutive acquisition for 10 periods of data in $10 \mathrm{~s}$ after intravenous administration. The total acquisition time was $30 \mathrm{~s}$, and each period of data was acquired at a regular interval of $30 \mathrm{~s}$, i.e., the total scanning time was 6 min. GFR was calculated by relevant softwares, with the simplified two compartment model (2C model) applied. The calculation formula of the model was: $\operatorname{Med}(\mathrm{t})=\mathrm{fCco}(\mathrm{t})+\Gamma^{*} \mathrm{Cco}(\mathrm{t})$. In this model, the pathway of contrast media was assumed as follows: renal cortex glomerular artery - glomerulus - renal medulla. Whereas, Med (t) was the time-dependent accumulation quantity of contrast media in renal medulla per unit volume, $\mathrm{f}$ was the capacitance percentage of the artery in renal medulla, Cco (t) was the time-dependent concentration of contrast media in renal cortex, $\Gamma$ was GFR per unit volume and * was convolution operator. GFR value was corrected by BSA, with the unit set as $\mathrm{ml} /\left(\min \cdot 1.73 \mathrm{~m}^{2}\right)$.

\subsection{The measurement of GFR by SPECT}

Philips Bright View SPECT scanner made by Philip was applied to the measurement of GFR. Patients were required to drink 300-500 $\mathrm{ml}$ of water $30 \mathrm{~min}$ ahead of getting an injection of ${ }^{99} \mathrm{mTc}-\mathrm{DTPA}$, and then empty their bladder before imaging, with height and weight recorded. The examinees were in the supine position, with the acquisition in the posterior view, with the renal regions and bladder placed in the visual field of the probe. After ${ }^{99} \mathrm{mTc}$-DTPA was infused via elbow vein in a pellet manner, SPECT renal dynamic imaging was performed immediately. The acquisition conditions were: LEHR, peak $140 \mathrm{keV}$, window width $20 \%$, matrix 64 $\times 64$, acquiring 30 frames at the speed of 1 frame $/ 2 \mathrm{~s}$ first, and then making an acquisition at the speed of 1 frame/60 s. The total acquisition time was $20 \mathrm{~min}$. Full and blank needle radioactive counts were measured and recorded before and after acquisition respectively to calculate radioactive counts in the human body. After acquisition, SPECT software kit was used to process images and calculate GFR of bilateral duplex kidneys by means of Gates method. GFR value was corrected by BSA, with the unit set as $\mathrm{ml} /\left(\mathrm{min} \cdot 1.73 \mathrm{~m}^{2}\right)$.

\subsection{Safety evaluation of contrast media}

The safety of contrast media was evaluated according to the modified Hartwing severity grading standards: ${ }^{[6]}$ Grade 1: mild adverse drug reactions and diseases, which were no need to be treated; Grade 2: transient impairment to patients, which needed some interventions such as drug withdrawal of suspicious drugs, without other treatments or (prolonged) hospitalization; Grade 3: transient impairment to patients, which needed some interventions such as drug withdrawal of suspicious drugs or other treatments, without (prolonged) hospitalization; Grade 4: patients had to be hospitalized or had to prolong the hospital stay for no less than $1 \mathrm{~d}$; Grade 5: patients needed intensive care; Grade 6: permanent impairment to patients; Grade 7: indirectly leading to a patient's death; Grade 8: directly leading to a patient's death.

\subsection{Statistical methods}

SPSS 22.0 software was applied to statistical processing and analysis, and the measurement data were represented by mean \pm standard deviation $(\bar{X} \pm \mathrm{s})$. The biases of GFRMRI and GFR-SPECT against Ccr value were considered as the mean differences of GFR-MRI and GFR-SPECT from Ccr value; the precision was the standard deviation (SD) of the bias; $30 \%$ and $50 \%$ accuracy was the percentage of GFR-MRI and GFR-SPECT in the range of $30 \%$ and $50 \%$ of Ccr value. Pearson correlation analysis and Bland-Altma were used to analyze the correlation and the consistency of GFR-MRI and GFR-SPECT with Ccr value. The difference $(p<.05)$ was of statistical significance.

\section{RESULTS}

\subsection{The biases of GFR-MKI and GFR-SPECT against Ccr value, precision and accuracy}

The bias of GFR-MRI against corrected Ccr value was higher than that of GFR-SPECT against corrected Ccr value. In addition, $30 \%$ and $50 \%$ accuracy of GFR-MRI was also higher than that of GFR-SPECT. The difference was of statistical significance $(p<.05)$. See Table 1 for details.

\subsection{The correlation analysis of GFR-MRI and GFR- SPECT to Ccr value}

Pearson correlation analysis was used to analyze the correlation of GFR-MRI and GFR-SPECT to Ccr value, and the results showed that both GFR-MRI and GFR-SPECT were positively correlated to Ccr value $(r=.821, .407, p<.05)$.

\subsection{The consistency analysis of GFR-MRI and GFR- SPECT with Cer value}

Bland-Altman was used to analyze the consistency of GFRMRI and GFR-SPECT with Ccr value. The result showed that $95 \%$ confidence interval of GFR-MRI was narrower than that of GFR-SPECT. See Table 2 for details.

\subsection{Safety evaluation}

The results of safety evaluation showed that there were 2 out of 70 cases of patients who developed Grade 1 adverse reaction, accounting for $2.86 \%$; patients had no Grade $2-8$ adverse reactions. 
Table 1. The biases of GFR-MRI and GFR-SPECT against Ccr value, precision and accuracy $(\mathrm{n}=70)$

\begin{tabular}{llllll}
\hline Indexes & $\begin{array}{l}\text { Measurement Value } \\
{\left[\mathbf{m l} /\left(\mathbf{m i n} \cdot \mathbf{1 . 7 3} \mathbf{~ m}^{\mathbf{2}}\right)\right]}\end{array}$ & Bias $\left[\mathbf{m l} /\left(\mathbf{m i n} \cdot \mathbf{1 . 7 3} \mathbf{~ m}^{\mathbf{2}}\right)\right]$ & $\begin{array}{l}\text { Precision } \\
{\left[\mathbf{m l} /\left(\mathbf{m i n} \cdot \mathbf{1 . 7 3} \mathbf{~ m}^{2}\right)\right]}\end{array}$ & $\begin{array}{l}\mathbf{3 0 \%} \text { Accuracy } \\
\mathbf{( \% )}\end{array}$ & $\begin{array}{l}\mathbf{5 0 \%} \text { Accuracy } \\
\mathbf{( \% )}\end{array}$ \\
\hline Ccr & $60.64 \pm 22.61$ & & & 66.74 & 73.26 \\
GFR-SPECT & $65.33 \pm 17.26$ & 4.69 & 23.58 & 83.25 & 93.47 \\
GFR-MRI & $50.43 \pm 22.67$ & -10.21 & 13.67 & \\
\hline Note. GFR-MRI: dynamic contrast-enhanced MRI; SPECT: single-photon emission computed tomography; Ccr: creatinine clearance rate; GFR: glomerular filtration rate.
\end{tabular}

Table 2. The consistency analysis of GFR-MRI and GFR-SPECT with Ccr value $\left[\mathrm{ml} /\left(\mathrm{min} \cdot 1.73 \mathrm{~m}^{2}\right), \mathrm{n}=70\right]$

\begin{tabular}{llll}
\hline Indexes & Upper Limit & Lower Limit & 95\% Confidence Interval \\
\hline GFR-SPECT & 55.13 & -40.36 & 95.49 \\
GFR-DCE-MRI & 43.20 & -19.15 & 62.35 \\
\hline
\end{tabular}

Note. GFR-MRI: dynamic contrast-enhanced MRI; SPECT: single-photon emission computed tomography; Ccr: creatinine clearance rate; GFR: glomerular filtration rate.

\section{Discussion}

Since the first renal transplantation was performed in clinical practice in 1954, renal transplantation has become a conventional treatment for end-stage renal disease, while surveillance of renal function and timely evaluation of the anatomical structure of transplant kidney are critical to effectively prolong the survival time of transplant kidney ${ }^{[7]}$ GFR is the most direct parameter which can evaluate renal function, and it is the most sensitive indicator in the evaluation of renal function. ${ }^{[8]}$ Currently, inulin clearance rate is still considered as the golden standard in GFR measurement, but it is only applicable to the experimental study due to its complicated operation. Nevertheless, the conventionally-used experimental method in the clinical application to measure creatinine and blood urea nitrogen levels is of a poor sensitivity. Therefore, inulin clearance rate is not an early indicator in the evaluation of GFR. In addition, it can not be used to evaluate the function of unilateral kidney. ${ }^{[9]}$ Currently, SPECT renal function test is an examination method which is commonly used to evaluate renal function in the clinical application and characterized by easy operation, safety and non-invasiveness. ${ }^{[10]}$ SPECT renal dynamic imaging has a good accuracy and repeatability in the evaluation of GFR in live transplant kidney donors. It has become a conventional examination to evaluate the donor's renal function before renal transplantation in the clinical application. ${ }^{[11]}$ However, SPECT is unable to present the anatomical information of renal blood vessels and urinary tract. ${ }^{[12]}$ In this research, the bias of GFR-SPECT against Ccr value is lower than that of GFR-DCE-MRI, but GFR-SPECT has a greater variation and a poorer consistency than GFR-DCE-MRI. The probable causes that lead to this result are that: 1) It is related with the corrected kidney depth. The corrected kidney depth adopted in the measurement of GFR by SPECT is an estimated value of the kidney depth in the normal anatomical position; how- ever, the depth of iliac fossa and the kidney depth have been changed after recipients are given renal transplantation, with a great variation. ${ }^{[13]} 2$ ) The transplant kidney is adjacent to iliac blood vessels and bladder, the contouring of renal ROI is prone to being affected in the measurement of GFR by SPECT, resulting in the error. ${ }^{[14]}$

DCE-MRI is a functional MRI imaging means which is applied early in the clinical application. It consecutively acquires dynamic T1 weighted images to contour TDC after injection of contrast media, and obtains hemodynamic parameters of tissue and lesion perfusion by utilizing different mathematic models. ${ }^{[15]}$ After DCE-MRI renal examination, the information on blood perfusion and filtration rate in unilateral kidney can be obtained by employing image processing to available proper models. ${ }^{[16]}$ In comparison with SPECT, DCE-MRI can clearly present two-dimension and three-dimension anatomical images of blood vessels and tissues peripheral to transplant kidney. It can effectively eliminate the effects brought by blood vessels and bladder in GR measurement of transplant kidney. ${ }^{[17]}$ In addition, DCE-MRI has a greater accuracy in contouring ROIs of renal cortex and medulla in transplant kidney. The calculation of GFR is not affected by the kidney depth, so that it can more accurately measure GFR. ${ }^{[18]}$ In this research, GFR-DCE-MRI has a high correlation and consistency with Ccr value, which conforms to the results in previous researches. ${ }^{[19]}$ However, the bias of GFR-DCE-MRI against Ccr is greater than that of SPECT against Ccr value, and the deviation may result from GFR calculation model adopted in the measurement by DCEMRI. The greatest advantage of the simplified $2 \mathrm{C}$ model is that it is not necessary to measure arterial input function, which is beneficial to the improvement of repeatability and stability for the measurement. However, this model assumes the input cavity is renal cortex, but the volume of proximal tubule in renal cortex is not taken into account, and the effects 
brought by the output of renal tubule in the output cavity of renal medulla and the exosmosis of contrast media to renal interstitium are ignored, resulting in the above-mentioned error.

The safety of contrast media is critical in the imaging examination. ${ }^{[20]}$ Some reports ${ }^{[21]}$ have indicated that contrast media of DCE-MRI, Gd-DOTA, has a low rate of adverse reactions and a high safety in the clinical application. In this research, there were only two cases of patients with Grade 1 adverse reaction (mild rash), which resulted from a small dose of Gd-DOTA and were improved without any treatments. It is indicated that a small dose of Gd-DOTA will not result in significant adverse reactions and the measurement of GFR by DCE-MRI has a high safety.

\section{REFERENCES}

[1] Halloran PF, Famulski KS, Reeve J. Molecular assessment of disease states in kidney transplant biopsy samples. Nat Rev Nephrol. 2016; 12(9): 534-548. PMid:27345248. https ://doi.org/10.1038/nr neph. 2016.85

[2] You S, Ma X, Zhang C, et al. Determination of single-kidney glomerular filtration rate (GFR) with CT urography versus renal dynamic imaging Gates method. Eur Radiol. 2017; 28(3): 18. PMid:28971295. https : //doi.org/10.1007/s00330-017-5 061-z

[3] Tsuda A, Ishimura E, Uedono H, et al. Comparison of the Estimated Glomerular Filtration Rate (eGFR) in Diabetic Patients, Non-Diabetic Patients and Living Kidney Donors. Kidney Blood Press Res. 2016; 41(1): 40-47. PMid:26836393. https : //doi .org/10.1159/0003 68545

[4] Ahmed AM, Qureshi WT, O'neal WT, et al. Incremental prognostic value of SPECT-MPI in chronic kidney disease: A reclassification analysis. J Nucl Cardiol. 2017; 25(5): 1658-1673. PMid:28050863. https ://doi.org/10.1007/s12350-016-0756-0

[5] Wanli Z, Jun T, Yudong Z, et al. Comparison of DCE-MRI renography, SPECT renography and endogenous creatinine clearance rate in kidney transplant recipients. Chinese Journal of Organ Transplantation. 2017; 38(5): 272-276.

[6] Rydberg DM, Holm L, Engqvist I, et al. Adverse Drug Reactions in a Tertiary Care Emergency Medicine Ward - Prevalence, Preventability and Reporting. PLoS One. 2016; 11(9): e0162948. PMid:27622270. https ://doi.org/10.1371/journal . pone. 0162948

[7] Ping C. Effects of mindfulness based cognitive therapy on quality of life and sleep status in end-stage renal disease patients with renal transplantation. China Medical Herald. 2017; 14(23): 148-151.

[8] Levey AS, Cassandra B, Inker LA. Glomerular filtration rate and albuminuria for detection and staging of acute and chronic kidney disease in adults: a systematic review. JAMA. 2015; 313(8): 837-846. PMid:25710660. https ://doi.org/10.1001/jama.2015.0602

[9] Fangyuan Z, Pengcheng L, Yu W. Comparison in the difference between GFR estimated by CKD-EPI formula and CCR. Zhejiang Journal of Preventive Medicine. 2017; 29(6): 646-648.

[10] Tran-Gia J, Schla( )q1 S, Lassmann M. Design and Fabrication of Kidney Phantoms for Internal Radiation Dosimetry Using 3D Printing Technology. J Nucl Med. 2016; 57(12): 1998-2005. PMid:27445291. https://doi.org/10.2967/jnumed.116.178046

\section{Conclusion}

In comparison with SPECT, DCE-MRI, as a new means in GFR measurement of transplant kidney, can be used to evaluate renal function of transplant kidney by measuring GFR and provide a new way to the evaluation of renal function of transplant kidney. In addition, unlike SPECT, DCE-MRI can better present the anatomical structure of transplant kidney, blood vessels and urinary tract with no obvious toxic and side effects. Therefore, DCE-MRI has a high applied value in the measurement of GFR to evaluate renal function in renal transplantation in clinical practice.

\section{CONFlicts OF InTEREST Disclosure}

The authors declare they have no conflicts of interest.
[11] Xiqiao X. Clinical value of SPECT renal dynamic imaging in the evaluation of renal function. Journal of Clinical Medicine in Practice. 2017; 21(5): 212-214.

[12] Jinlin D, Jian L, Weimin J, et al. Research on the correlation of TCM syndrome differentiation in hypertension to GFR-SPECT and eGFR. Chinese Journal of Integrative Medicine on Cardio-/Cerebrovascuiar Disease. 2016; 14(24): 2871-2873.

[13] Rui Z, Huanhuan W, Feng D, et al. Evaluation of early kidney injury induced by chronic B virus hepatitis using dynamic contrast enhanced MRI renogram. Radiologic Practice. 2016; 31(11): 1071-1075.

[14] Pingping H, Ling L, Jie L, et al. Effect of Kidney Depth in Glomerular Filtration Rate Measured by Kidney Dynamic Imaging. China Medical Devices. 2017; 32(6): 66-69.

[15] Aibo W, Jie B. The principle and application in clinic of dynamic contrast enhancement magnetic resonance imaging. Journal of China Clinic Medical Imaging. 2016; 27(6): 435-438.

[16] Eikefjord E, Andersen E, Hodneland E, et al. Quantification of Single-Kidney Function and Volume in Living Kidney Donors Using Dynamic Contrast-Enhanced MRI. AJR Am J Roentgenol. 2016; 207(5): 1022-1030. PMid:27557401. https ://doi.org/10.221 4/AJR. 16.16168

[17] Rui Z, Huanhuan W, Feng D, et al. Evaluation of early kidney injury induced by chronic B virus hepatitis using dynamic contrast enhanced MRI renogram. Radiologic Practice. 2016; 31(11): 1071-1075.

[18] Peperhove M, Vo Chieu VD, Jang MS, et al. Assessment of acute kidney injury with T1 mapping MRI following solid organ transplantation. Eur Radiol. 2018; 28(1): 1-7. PMid:28710580. https : //doi.org/10.1007/s00330-017-4943-4

[19] Jiang K, Tang H, Mishra PK, et al. Measurement of Murine Single-Kidney Glomerular Filtration Rate Using Dynamic ContrastEnhanced MRI. Magn Reson Med. 2017; 79(6): 2935-2943. PMid:29034514. https : //doi.org/10.1002/mrm. 26955

[20] Kanakia S, Toussaint J, Kukarni P, et al. Safety and Efficacy of A High Performance Graphene-Based Magnetic Resonance Imaging Contrast Agent for Renal Abnormalities. Graphene Technol. 2016; 1(1/4): 1-12. PMid:28261636. https://doi.org/10.1007/s411 27-016-0001-2

[21] Yanhui Z, Hongyan Z, Hailu Z, et al. A New Gd-Based T2-Weighted Magnetic Resonance Imaging Contrast Agent: Preparation and Application in Stem Cell Imaging. Chinese Journal of Magnetic Resonance. 2017; 34(3): 302-310. 